\title{
PARAMETRIC VIBRATION OF MECHANICAL SYSTEM WITH SEVERAL DEGREES OF FREEDOM UNDER THE ACTION OF ELECTROMAGNETIC FORCE
}

\author{
NGUYEN VAN DAO \\ Department of Methemathics and Physics Polytechnic Institute, Hanoi
}

(This paper has been published in:

Proceedings of Vibration Problems, 14, 1, pp.85-94, 1973

Institute of Fundamental Technological Research, Polish Academy of Sciences)

\section{SYSTEMS WITH $n$ DEGREES OF FREEDOM}

Let us consider a vibrating system with $n$ degrees of freedom which consists of a weightless cantilever beam carrying $n$ concentrated masses $m_{1}, m_{2}, \ldots, m_{n}$ (Fig. 1). The elastic elements of the vibrating system have stiffness $k_{1}, k_{2}, \ldots, k_{n}$.

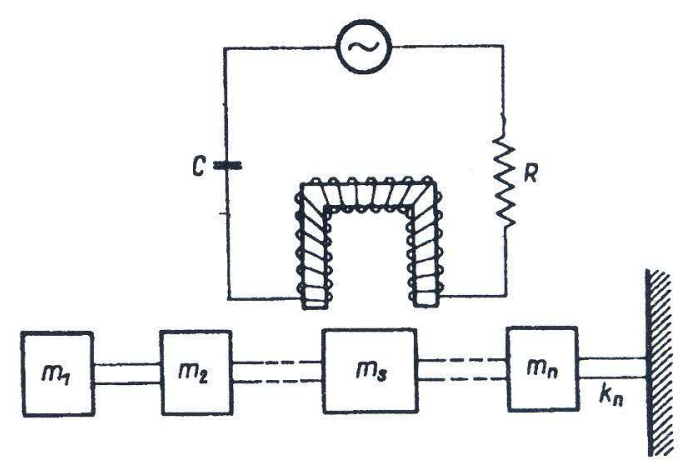

Fig. 1

Supposing that some $s^{\text {th }}$ mass is subjected to electromagnetic force, the differential equations of motion of the system considered can be written, in accordance with [1] in the form:

$$
\begin{aligned}
& \frac{d}{d t}(L \dot{q})+R \dot{q}+\frac{1}{C} q=E \sin \nu t, \\
& m_{1} \ddot{x}_{1}+k_{1}\left(x_{1}-x_{2}\right)=-h_{1} \dot{x}_{1}-\beta_{1}\left(x_{1}-x_{2}\right)^{3}, \\
& m_{2} \ddot{x}_{2}+k_{1}\left(x_{2}-x_{1}\right)+k_{2}\left(x_{2}-x_{3}\right)=-h_{2} \dot{x}_{2}-\beta_{1}\left(x_{2}-x_{1}\right)^{3}-\beta_{2}\left(x_{2}-x_{3}\right)^{3},
\end{aligned}
$$




$$
\begin{aligned}
& \begin{array}{lll}
\cdots & \ldots & \ldots
\end{array} \\
& m_{s} \ddot{x}_{s}+k_{s-1}\left(x_{s}-x_{s-1}\right)+k_{s}\left(x_{s}-x_{s+1}\right)=-h_{s} \dot{x}_{s}-\beta_{s-1}\left(x_{s}-x_{s-1}\right)^{3} \\
& -\beta_{s}\left(x_{s}-x_{s+1}\right)^{3}+\frac{1}{2} \dot{q}^{2} \frac{\partial L}{\partial x_{s}}, \\
& m_{n} \ddot{x}_{n}+k_{n-1}\left(x_{n}-x_{n-1}\right)+k_{n} x_{n}=-h_{n} \dot{x}_{n}-\beta_{n-1}\left(x_{n}-x_{n-1}\right)^{3}-\beta_{n} x_{n}^{3} .
\end{aligned}
$$

We assume that

$$
L=L\left(x_{s}\right)=L_{0}\left(1-\alpha_{1} x_{s}+\alpha_{2} x_{s}^{2}\right),
$$

and that the friction forces and the non-linear terms in (1.1) are small with respect to the remaining terms. Then, Eqs. (1.1) can be rewritten as:

$$
\begin{aligned}
& L_{0} \ddot{q}+\frac{1}{C} q=E \sin \nu t-\mu\left[L_{0} \ddot{q}\left(-\alpha_{1} x_{2}+\alpha_{2} x_{s}^{2}\right)+\dot{q} L_{0}\left(-\alpha_{1} \dot{x}_{s}+2 \alpha_{2} x_{s} \dot{x}_{x}\right)\right], \\
& m_{1} \ddot{x}_{1}+k_{1}\left(x_{1}-x_{2}\right)=\mu F_{1}, \\
& m_{2} \ddot{x}_{2}+k_{1}\left(x_{2}-x_{1}\right)+k_{2}\left(x_{2}-x_{3}\right)=\mu F_{2}, \\
& \quad \ldots \quad \ldots \quad \ldots \quad \ldots \\
& m_{s} \ddot{x}_{s}+k_{s-1}\left(x_{s}-x_{s-1}\right)+k_{s}\left(x_{s}-x_{s+1}\right)=-\frac{1}{2} \dot{q}^{2} L_{0} \alpha_{1}+\mu_{s} F_{s}, \\
& \quad \ldots \quad \ldots \quad \ldots \quad \ldots \\
& m_{n} \ddot{x}_{n}+k_{n-1}\left(x_{n}-x_{n-1}\right)+k_{n} x_{n}=\mu F_{n}
\end{aligned}
$$

where

$$
\begin{aligned}
& \mu F_{1}=-h_{1} \dot{x}_{1}-\beta_{1}\left(x_{1}-x_{2}\right)^{3}, \\
& \mu F_{2}=-h_{2} \dot{x}_{2}-\beta_{1}\left(x_{2}-x_{1}\right)^{3}-\beta_{2}\left(x_{2}-x_{3}\right)^{3}, \\
& \text {. } \\
& \mu F_{s}=-h_{s} \dot{x}_{s}+\dot{q}^{2} L_{0} \alpha_{2} x_{s}-\beta_{s-1}\left(x_{s}-x_{s-1}\right)^{3}-\beta_{s}\left(x_{s}-x_{s+1}\right)^{3} \text {, } \\
& \mu F_{n}=-h_{n} \dot{x}_{n}-\beta_{n-1}\left(x_{n}-x_{n-1}\right)^{3}-\beta_{n} x_{n}^{3} .
\end{aligned}
$$

We suppose that the characteristic equation of the homogeneous system

$$
\begin{aligned}
& m_{1} \ddot{x}_{1}+k_{1}\left(x_{1}-x_{2}\right)=0, \\
& m_{2} \ddot{x}_{2}+k_{1}\left(x_{2}-x_{1}\right)+k_{2}\left(x_{2}-x_{3}\right)=0, \\
& \ldots \quad \ldots \quad \ldots \quad \ldots \\
& m_{n} \ddot{x}_{n}+k_{n-1}\left(x_{n}-x_{n-1}\right)+k_{n} x_{n}=0,
\end{aligned}
$$

has no multiple roots and that its roots $\omega_{1}, \ldots, \omega_{n}$ are linearly independent. Then, to study the system (1.2), we shall analyze its particular solution corresponding to the onefrequency regime of vibrations [2]. To that end, we introduce the normal coodinates $\xi_{1}, \ldots, \xi_{n}$ by means of the formulae:

$$
x_{s}=\sum_{\sigma=1}^{n} c_{s}^{(\sigma)} \xi_{\sigma}, \quad s=1,2, \ldots, n
$$


where $c_{s}^{(\sigma)}$ is algebraic supplement of the element placed in the $s$-th column and the lasrt line of the characteristic determinant of the system (1.4).

We can easily verify that the normal coordinates $\xi_{1}, \ldots, \xi_{n}$, satisfy the following equations:

$$
\begin{aligned}
& L_{0} \ddot{q}+\frac{1}{C} q=E \sin \nu t-\mu F_{0}\left(\dot{q}, \ddot{q}, \sum_{\sigma=1}^{n} c_{s}^{(\sigma)} \xi_{\sigma}, \sum_{\sigma=1}^{n} c_{s}^{(\sigma)} \dot{\xi}_{\sigma}\right), \\
& \ddot{\xi}_{j}+\omega_{j}^{2} \xi_{j}=\mu \Phi_{j}\left(\sum_{\sigma=1}^{n} c_{1}^{(\sigma)} \xi_{\sigma}, \ldots, \sum_{\sigma=1}^{n} c_{n}^{(\sigma)} \xi_{s}, \sum_{\sigma=1}^{n} c_{1}^{(\sigma)} \dot{\xi}_{s}, \ldots, \sum_{\sigma=1}^{n} c_{n}^{(\sigma)} \dot{\xi}_{\sigma}, \dot{q}\right) .
\end{aligned}
$$

Here

$$
\begin{aligned}
& F_{0}\left(\dot{q}, \ddot{q}, x_{s}, \dot{x}_{s}\right)=L_{0} \ddot{q}\left(-\alpha_{1} x_{s}+\alpha_{2} x_{s}^{2}\right)+L_{0} \dot{q} \dot{x}_{s}\left(-\alpha_{1}+2 \alpha_{2} \alpha_{s}\right)+R \dot{q}, \\
& \Phi_{j}=\frac{1}{M_{j}} \sum_{k=1}^{n} c_{k}^{(j)} F_{k}-\frac{\alpha_{1} L_{0} \dot{q}^{2}}{2 \mu M_{j}} c_{s}^{(j)} \\
& M_{j}=\sum_{i=1}^{n} m_{i} c_{1}^{2(j)}
\end{aligned}
$$

In the first approximation, the investigation of one-frequency regime in the system considered can be reduced to a study of two equations: the first of (1.6) and one of remaining $n$ equations. The choice of the appropriate equation depends on the value of natural frequency $\omega$ in the neighbourhood of which the parametric vibrations are examined. Supposing that the frequency $\nu$ of external force is near the nartural frequency $\omega_{j}$. Then we shall investigate the equations:

$$
\begin{aligned}
& L_{0} \ddot{q}+\frac{1}{C} q=E \sin \nu t-\mu F_{0}^{*}, \\
& \ddot{\xi}_{j}+\omega_{j}^{2} \xi_{j}=-\mu h^{*} \dot{\xi}_{j}-\mu \beta^{*} \xi_{j}^{3}-\frac{\alpha_{1} L_{0} c_{s}^{(j)}}{2 M_{j}} \dot{q}^{2}+\frac{\mu}{M_{j}} L_{0} \alpha_{2} c_{s}^{2(j)} \dot{q}^{2} \xi_{j},
\end{aligned}
$$

where

$$
\begin{gathered}
\mu h^{*}=\frac{1}{M_{j}} \sum_{s=1}^{n} h_{s} c_{s}^{2(j)} \\
\mu \beta^{*}=\frac{1}{M_{j}}\left[\beta_{1} c_{1}^{(j)}\left(c_{1}^{(j)}-c_{2}^{(j)}\right)^{3}+\beta_{1} c_{1}^{(j)}\left(c_{2}^{(j)}-c_{1}^{(j)}\right)^{3} \beta_{2} c_{2}^{(j)}\left(c_{2}^{(j)}-c_{3}^{(j)}\right)^{2}+\ldots\right. \\
+\beta_{s-1} c_{s}^{(j)}\left(c_{s}^{(j)}-c_{s-1}^{(j)}\right)^{3}+\beta_{s} c_{s}^{(j)}\left(c_{s}^{(j)}-c_{s+1}^{(j)}\right)+\ldots \\
\left.+\beta_{n-1} c_{n}^{(j)}\left(c_{n}^{(j)}-c_{n-1}^{(j)}\right)^{3}+\beta_{n} c_{n}^{4(j)}\right] \\
\mu F_{0}^{*}=L_{0} \ddot{q}\left(-\alpha_{1} c_{s}^{(j)} \xi_{j}+\alpha_{2} c_{s}^{2(j)} \xi_{j}^{2}\right)+L_{0} \dot{q} c_{s}^{(j)} \dot{\xi}_{j}\left(-\alpha_{1}+2 \alpha_{2} c_{s}^{(j)} \xi_{j}\right) .
\end{gathered}
$$

The remaining $n-1$ normal coordinates $\xi_{1}, \ldots, \xi_{j-1}, \xi_{j+1}, \ldots, \xi_{n}$ are far from the resonance, their vibration will be small in comparison with the resonant vibration considered of the coordinate $\xi_{j}$, and in the first approximation they may be disregarded.

Equations (1.7) describing the one-frequency regime of vibrations have the same structure as the equations of motion of the system with single degree of freedom [1]. This gives 
reason to expect that in each resonant region the same peculiarities of motion will be displayed were found in the system with a single degree of freedom.

Introducing the notations

$$
\begin{aligned}
h & =\frac{h^{*}}{\omega_{j}}, \quad \tau=\omega_{j} t, \quad \beta=\frac{\beta^{*}}{\omega_{j}^{2}}, \quad \alpha_{1}^{*}=\frac{\alpha_{1} L_{0} c_{s}^{(j)}}{2 M_{j}}, \\
\alpha_{2}^{*} & =\frac{1}{M_{j}} L_{0} \alpha_{2} c_{s}^{2(j)}, \quad \Omega_{j}=\frac{\Omega_{0}}{\omega_{j}}, \quad \Omega_{0}^{2}=\frac{1}{L_{0} C}, \quad e_{j}=\frac{L_{0}}{E_{0} \omega_{j}^{2}}, \quad \gamma_{j}=\frac{\gamma}{\omega_{j}},
\end{aligned}
$$

Eqs. (1.7) assume the form:

$$
\begin{aligned}
q^{\prime \prime}+\nabla_{j}^{2} q & =e_{j} \sin \gamma_{j} \tau-\frac{\mu}{L_{0} \omega_{j}^{2}} F_{0}^{*}, \\
\xi_{j}^{\prime \prime}+\xi_{j} & =-\mu h \xi_{j}^{\prime}-\mu \beta \xi_{j}^{3}-\alpha_{1}^{*} q^{2}+\mu \alpha_{2}^{*} q^{2} \xi_{j} .
\end{aligned}
$$

Now, we transform the system (1.9) by means of the formulae:

$$
\begin{aligned}
q & =e_{j}^{*} \sin \gamma_{j} \tau+B \sin \varphi \\
q^{\prime} & =\gamma_{j} e_{j}^{*} \cos \gamma_{j} \tau+\Omega_{j} B \cos \varphi, \\
\xi_{j} & =-b-\frac{b}{1-4 \gamma_{j}^{2}} \cos 2 \gamma_{j} \tau+A_{j} \sin \theta_{j}, \\
\xi_{j}^{\prime} & =\frac{2 \gamma_{j} b}{1-4 \gamma_{j}^{2}} \sin 2 \gamma_{j} \tau+A_{j} \gamma_{j} \cos \theta_{j},
\end{aligned}
$$

where

$$
e_{j}^{*}=\frac{e_{j}}{\Omega_{j}^{2}-\gamma_{j}^{2}}, \quad b=\frac{\alpha_{j}^{*} \gamma_{j}^{2} e_{j}^{* 2}}{2}, \quad \varphi=\Omega_{j} \tau, \quad \theta_{j}=\gamma_{j} \tau+\psi_{j}
$$

The transformed equations have the form:

$$
\begin{aligned}
\Omega_{j} \frac{d B}{d \tau} & =-\frac{\mu}{L_{0} \omega_{j}} F_{0}^{*} \cos \varphi \\
\Omega_{j} B \frac{d \Phi}{d \tau} & =\frac{\mu}{L_{0} \omega_{j}^{2}}=F_{0}^{*} \sin \varphi \\
\gamma_{j} \frac{d A_{j}}{d \tau} & =-A_{j}\left(1-\gamma_{j}^{2}\right) \sin \theta_{j} \cos \theta_{j}-\mu S \cos \theta_{j}+\ldots, \\
A_{j} \gamma_{j} \frac{d \psi_{j}}{d \tau} & =A_{j}\left(1-\gamma_{j}^{2}\right) \sin ^{2} \theta_{j}+\mu S \sin \theta_{j}+\ldots, \\
S & =h \xi_{j}^{\prime}+\beta \xi_{j}^{3}-\alpha_{2}^{*} q^{\prime 2} \xi
\end{aligned}
$$

where the non-written terms vanish when $B=0$.

We suppose that $\gamma_{j}$ is in the neighbourhood of 1 and that $\gamma_{j}$ and $\Omega_{j}$ are linearly indepenent. Then, in the first approximation the solution of the system (1.9) satisfies the equations obtained from (1.9) by averaging in time its right-hand part

$$
[1+0(\mu)] \frac{d B}{d \tau}=-\mu \frac{R}{2} B
$$




$$
\begin{aligned}
\frac{d \Phi}{d \tau} & =G\left(B, \Phi, A_{j}, \psi_{j}\right), \\
\gamma_{j} \frac{d A_{j}}{d \tau} & =-\mu \frac{h}{2} \gamma_{j} A_{j}+\frac{\mu}{2} c_{1} A_{j} \sin 2 \psi_{j}+\ldots, \\
\gamma_{j} A_{j} \frac{d \psi_{j}}{d \tau} & =\frac{1}{2}\left(1-\gamma_{j}^{2}+\mu \Delta\right) A_{j}+\frac{3}{8} \mu \beta A_{j}^{2}+\frac{\mu}{2} c_{1} A_{j} \cos 2 \psi_{j}+\ldots
\end{aligned}
$$

where

$$
\begin{aligned}
c_{1} & =\frac{q}{2}+\frac{3 \beta b^{2}}{1-4 \gamma_{j}^{2}}, \\
A & =q+3 \beta b^{2}+\frac{3}{2} \beta \frac{b^{2}}{\left(1-4 \gamma_{j}^{2}\right)^{2}}, \\
b & =\frac{\alpha_{j}^{*} \gamma_{j}^{2} c_{j}^{* 2}}{2}, \quad q=\frac{\alpha_{2}^{*}}{2} \gamma_{j}^{2} e_{j}^{* 2} .
\end{aligned}
$$

Since $B \rightarrow 0$ when $t \rightarrow \infty$, then below we shal take into account only the equations:

$$
\begin{aligned}
\gamma_{j} \frac{d A_{j}}{d \tau} & =-\mu \frac{h}{2} \gamma_{j} A_{j}+\frac{\mu}{2} c_{1} A_{j} \sin 2 \psi_{j}, \\
\gamma_{j} A_{j} \frac{d \psi_{j}}{d \tau} & =\frac{1}{2}\left(1-r_{j}^{2}+\mu \Delta_{1}\right) A_{j}+\frac{3}{8} \mu \beta A_{j}^{3}+\frac{\mu}{2} c_{1} A_{j} \cos 2 \psi_{j},
\end{aligned}
$$

from which we obtain the amplitude $A_{j}$ of vibrations:

$$
A_{j}^{2}=\frac{4}{3 \beta}\left(\frac{\gamma_{j}^{2}-1}{\mu}-\Delta \pm \sqrt{c_{1}^{2}-h^{2} \gamma_{j}^{2}}\right),
$$

and the phase

$$
\sin 2 \psi_{j}=\frac{h}{c_{1}} \gamma_{j}, \quad \cos 2 \psi_{j}=\mp \frac{1}{c_{1}} \sqrt{c_{1}^{2}-h^{2} \gamma_{j}^{2}} .
$$

Equations (1.12)-(1.15) are different from the corresponding ones in the system with a single degree of freedom [1] only by the values of the constant coefficients. The method used enabled us to reduce the more complicated problem to the whole complex of $n$ problems of the type considered earlier. In spite of this, in the first approximation each of such problems can be investigated independently of the others, because according to the conditions of the problem, the resonant processes cannot be developed at the same time in more than on resonant region.

The stability of stationary regimes of vibrations may be found by analysing Eqs. (1.12). The criteria of stability formed in [1] are:

and

$$
\begin{gathered}
\frac{\partial W}{\partial A_{j}}>0 \text { for } A_{j} \neq 0 \\
W=\left(\frac{3}{4} \mu \beta A_{j}^{2}+1-\gamma_{j}^{2}+\mu \Delta\right)^{2}-\mu^{2}\left(c_{1}^{2}-h^{2} \gamma_{j}^{2}\right),
\end{gathered}
$$

$$
\mu^{2}\left(h^{2} \gamma_{j}^{2}-c_{1}^{2}\right)+\left(\gamma_{j}^{2}-1-\mu \Delta\right)^{2}>0 \text { for } A_{j}=0 .
$$

The study made in [1] concerning the stability of stationary regimes of motion will be suitable for the character of resonant processes described by Eqs. (1.12) in qualitative 
relation. This removes the necessity of analysis in detail the criteria of stability. Here we note only that for very slow change of frequency $\nu$ in the system considered, $n$ resonant peaks corresponding to the values $\nu=\omega_{1}\left(\gamma_{1}=1\right), \nu=\omega_{2}\left(\gamma_{2}=1\right) \ldots$ are observed (Fig. 2).

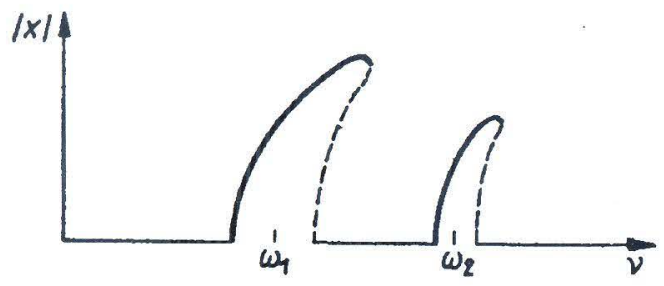

Fig. 2

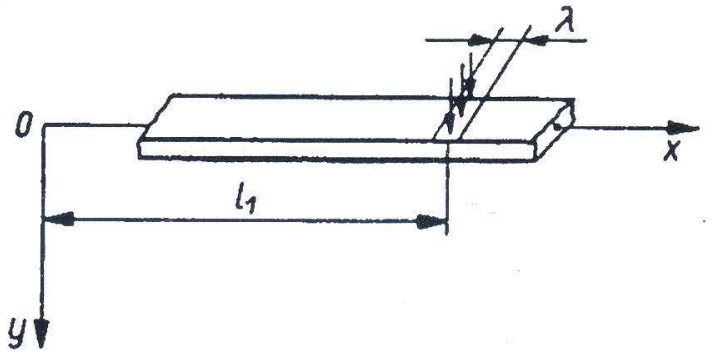

Fig. 3

\section{PARAMETRIC RESONANCE IN A SYSTEM WITH INFINITE NUMBER OF DEGREES OF FREEDOM}

We investigate in the Cartesian coordinates $x, y, z$ a prismatic beam with length $\ell$ whose cross-section is symmetrical with respect to two mutually perpendicular axes. We assume that the axis of the beam in the underformed state coincides with the axis $x$ and that the symmetrical axes are parallel to the axes $y$ and $z$ (Fig. 3).

The beam under certain conditions of strengthning of its end is subjected to the action of electromagnetic force which is $\ell_{1}$ distant from the origin of the coordinates and directed to the axis $y$. We assume that the inductance $L$ is a function of distance $y_{1}=y\left(\ell_{1}, t\right)$,

$$
L=L\left(y_{1}\right)=L_{0}\left(1-\alpha_{1} y_{1}+\alpha_{2} y_{1}^{2}\right),
$$

and therefore the electromagnetic force depends on the location of the electromagnet and on the vibrations of the beam, and has intensity $\frac{1}{2} \dot{q}^{2} \frac{\partial L}{\partial \ell_{1}}$.

We assume that the material of the beam follows the law [3]

$$
\sigma_{x}=f\left(\varepsilon_{x}\right)=E\left(1-d E^{2} \varepsilon_{x}^{2}\right) \varepsilon_{x},
$$

where $\sigma_{x}$ is the longitudinal force and $\varepsilon_{x}$ is the longitudinal elongation. Then, the equation of motion of the beam is:

$$
\frac{\partial^{2} M}{\partial x^{2}}=-\rho \frac{\partial^{2} y}{\partial t^{2}}-H \frac{\partial y}{\partial t}+P(x, t)
$$

where $\rho$ is the intensity of mass of the beam, $y=y(x, t)$-the deflection, $P(x, t)$-the intensity of external load, $M(x, t)$-the bending moment:

$$
M=\iint f\left(y \frac{\partial^{2} y}{\partial x^{2}}\right) y d y d z=E \iint\left[1-d E^{2} y^{2}\left(\frac{\partial^{2} y}{\partial x^{2}}\right)^{2}\right] y^{2} \frac{\partial^{2} y}{\partial x^{2}} d y d z .
$$

Substituting this expression into (2.2), we obtain:

$$
\rho \frac{\partial^{2} y}{\partial t^{2}}+E J \frac{\partial^{4} y}{\partial x^{4}}=3 d E^{3} J_{1}\left[\frac{\partial^{4} y}{\partial x^{4}} \frac{\partial^{2} y}{\partial x^{2}}+2\left(\frac{\partial^{3} y}{\partial x^{3}}\right)^{2}\right] \frac{\partial^{2} y}{\partial x^{2}}-H \frac{\partial y}{\partial t}+P(x, t),
$$


where,

$$
J_{1}=\iint y^{4} d y d z, \quad J=\iint y^{2} d y d z .
$$

We assume that the non-linear terms and the terms characterizibng friction are small in comparison with the linear terms. Then the equation of motion of the system considered can be represented in the form:

$$
\begin{gathered}
\ddot{q}+\Omega_{0}^{2} q=e \sin \nu t+\mu F_{1}\left(y_{1}, \frac{\partial y_{1}}{\partial t}, \dot{q}, \ddot{q}\right), \\
\frac{\partial^{2} y}{\partial t^{2}}+b^{2} \frac{\partial^{4} y}{\partial x^{4}}=\mu F_{2}
\end{gathered}
$$

where

$$
\begin{aligned}
\Omega_{0}^{2} & =\frac{1}{L_{0} C}, \quad e=\frac{E}{L_{0}}, \quad b=\frac{E J}{\rho}, \quad \beta=\frac{3 d E^{3} J_{1}}{\rho}, \\
\mu F_{1} & =-\frac{R}{L_{0}} \dot{q}+\alpha_{1}\left(\dot{q} \frac{\partial y_{1}}{\partial t}+y_{1} \ddot{q}\right)-\alpha_{2}\left(2 y_{1} \dot{q} \frac{\partial y_{1}}{\partial t}+\ddot{q} y_{1}^{2}\right), \\
\mu F_{2} & =-\frac{H}{\rho} \frac{\partial y}{\partial t}+\beta\left[\frac{\partial^{4} y}{\partial x^{4}} \frac{\partial^{2} y}{\partial x^{2}}+2\left(\frac{\partial^{3} y}{\partial x^{3}}\right)^{2}\right] \frac{\partial^{2} y}{\partial x^{2}}+\frac{1}{\rho} P(x, t) .
\end{aligned}
$$

The external load $P(x, t)$ has the form:

$$
P(x, t)= \begin{cases}0 & \text { for } 0 \leqslant x<\ell_{1}-\frac{\lambda}{2}, \\ \frac{L_{0} \alpha_{2}}{\lambda} \dot{q}^{2} y_{1}-\frac{L_{0} \alpha_{1}}{2 \lambda} \dot{q}^{2} & \text { for } \ell_{1}-m \frac{\lambda}{2} \leqslant x \leqslant \ell_{1}+\frac{\lambda}{2}, \\ 0 & \text { for } \ell_{1}+\frac{\lambda}{2}<x \leqslant \ell,\end{cases}
$$

where $\lambda$ is the length of that element of the beam is directly subjected to the action of electromagnetic force.

To solve the system $(2.3)$, we note first that the generative equations $(\mu=0)$

$$
\ddot{q}+\Omega_{0}^{2} q=e \sin \nu t, \quad \frac{\partial^{2} y}{\partial t^{2}}+b^{2} \frac{\partial^{4} y}{\partial x^{4}}=0
$$

have the solution:

$$
\begin{aligned}
& q=e^{*} \sin \nu t+B \sin \varphi \\
& y=\sum_{n=1}^{\infty} X_{n}(x) c_{n} \cos \left(\frac{m_{n}^{2}}{\ell^{2}} b t+\gamma_{n}\right),
\end{aligned}
$$

where $B, \Phi, c_{n}, \gamma_{n}$ are arbitrary constants, $X_{n}$ are the eigenfunctions which define the natural modes of vibrations of the beam and depend on the boundary conditions.

Equations (2.3) are different from the corresponding ones of the systems (2.6) only by small terms $\mu F_{1}, \mu F_{2}$. Consequently, it is natural to propose the following form of solution of the system (2.3):

$$
q=e^{*} \sin \nu t+B \sin \varphi, \quad y=\sum_{n=1}^{\infty} X_{n}(x) s_{n}(t)
$$


where $\varphi=\Omega_{0} t+\Phi$ and $B, \Phi, s_{n}$ are functions of time.

Now, instead of determining the functions $q$ and $y$, we determine the functions $B, \Phi, s_{n}$. To find the different equations for these variables, we represent $F_{2}$ in the form of a series:

$$
F_{2}=\sum_{n=1}^{\infty} X_{n} V_{n}\left(s_{1}, s_{2}, \ldots, \dot{s}_{1}, \dot{s}_{2}, \ldots, t\right) .
$$

To seek the functions of time $V_{n}$, we multiply both sides of the equality (2.9) by $X_{i}$, and integrate the result over the total length of the beam; due to the orthogonality of the eigenfunctions there remains only term on the right-hand side which corresponds to the number $n$, so that

$$
V_{n}=\int_{0}^{\ell} F_{2} X_{n} d x / \int_{0}^{\ell} X_{n}^{2} d x .
$$

Substituting (2.8), (2.9) into (2.3) and equating the coefficients $X_{n}$, we arrive at:

$$
\begin{aligned}
\ddot{q}+\Omega_{0}^{2} q & =e \sin \nu t+\mu F_{1}, \\
\ddot{s}_{n}+\omega_{n}^{2} s_{n} & =\mu r_{n} L_{0} \alpha_{2} \dot{q}^{2} S_{n}+\mu K_{n}\left(s_{1}, s_{2}, \ldots, \dot{s}_{1}, \dot{s}_{2}, \ldots, t\right),
\end{aligned}
$$

where $K_{n}$ are polynomials of third degree, relatively of $s_{1}, s_{2}, \ldots$

We consider now parametric resonance when the frequency of the electric circuit $\nu$ is in the neighbourhood of $\omega_{j}$ assuming that the natural frequencies $\omega_{1}, \omega_{2}, \ldots$ are independent. Then we retain in (1.1) only the coordinates $s_{j}$. The remaining coordinates $s_{1}, \ldots, s_{j-1}, s_{j+1}, \ldots$ are far from the resonance and their values will be small in comparison with $s_{j}$ and in the first approximation we can disregard them. Thus, following the expressions (2.4), (2.8), we have:

$$
\mu F_{2}=-\frac{H}{\rho} \dot{s}_{j} X_{j}+\beta\left[X_{j}^{I V} X_{j}^{\prime \prime 2}+2 X_{j}^{\prime \prime \prime} X_{j}^{\prime \prime}\right] s_{j}^{3}+\frac{P}{\varrho}
$$

Therefore, from (2.10), (2.11) we obtain the equations for $q, s_{n}$ :

$$
\begin{aligned}
\ddot{q}+\Omega_{0} q & =e \sin \nu t+\mu F_{1}, \\
\ddot{s}_{j}+\omega_{j}^{2} s_{j} & =-\frac{H}{\rho} \dot{s}_{j}+\beta a_{j} s_{j}^{3}+b_{j} \dot{q}^{2} s_{j}-c \dot{q}^{2},
\end{aligned}
$$

where

$$
\begin{aligned}
& a_{j}=\int_{0}^{\ell}\left(X_{j}^{I V} X_{j}^{\prime \prime 2}+X_{j}^{\prime \prime 2} X_{j}^{\prime \prime}\right) X_{j} d x / \int_{0}^{\ell} X_{j}^{2} d x, \\
& b_{j}=L_{0} \alpha_{2} X_{j}\left(\ell_{1}\right) \int_{\ell_{1}-\frac{\lambda}{2}}^{\ell_{1}+\frac{\lambda}{2}} X_{j} d x / \lambda \rho \int_{0}^{\ell} X_{j}^{2} d x,
\end{aligned}
$$




$$
c_{j}=\frac{L_{0} \alpha_{1}}{2} \int_{\ell_{1}-\frac{\lambda}{2}}^{\ell_{1}+\frac{\lambda}{2}} X_{j} d x / \lambda \rho \int_{0}^{\ell} X_{j}^{2} d x .
$$

It is easily seen that the system of Eqs. (2.12) is the complete analogy of the differential equations of vibrations of a system with single degree of freedom. To avoid repetition, we shall refer below to the paper [1], where the problem of construction of a solution of the system of equations of the form (2.12) is considered in detail.

Thus, following the results of [1], we conclude that when the frequency $\nu$ of an electric circuit is near to $\omega_{1}$, then the beam considered vibrates strongly with frequency $\nu$ (parametric resonance). This type of resonance takes place also when the frequency $\nu$ is near to $\omega_{2}, \omega_{3}, \ldots$ However, it must be emphasized that in the system with distributed parameters the vibrations with the lowest frequency $\left(\omega_{1}\right)$ play the main role.

Some experiments were performed with beams and systems of several degrees of freedom. The experimental results in the cases considered were in good agreement with the theoretical results. This fact testifies to the acceptability of the limitations used is the problem and shows that the approximate solutions found by using the assumption concerning the one-frequency regime of vibrations in the regions of resonance can be adopted for practical purpose.

For the cantilever beam with parameters $E=2 \cdot 10^{7} \mathrm{~N} / \mathrm{cm}^{2}, J=16 \cdot 10^{-3} \mathrm{~cm}^{4}$, $\varphi=10^{-4} \mathrm{~N} \cdot \mathrm{s}^{2} / \mathrm{cm}^{2}, \ell=46 \mathrm{~cm}$; therefore, $\omega_{1}=14.8, \omega_{2}=93.7$, strong vibrations with frequency of electric circuit $\nu$ when $\nu$ is in the region $13.5-14.3 \mathrm{~Hz} \ldots$, were very small. For the same beam, but when $\ell=58 \mathrm{~cm}$ and therefore $\omega_{1}=26.3$, substantial parametric resonance when $\nu$ is the region $27.1-29.4 \mathrm{~Hz}$ was observed.

\section{REFERENCES}

1. Nguyen Van Dao, On the phenomenons of parametric resonance of a non-linear vibrator under the action of electromagnetic force, Vibr. Probl. 13 (3) (1972).

2. Н. Н. Боголюбов, Ю. А. Митропольский, Асимптотические методы в теории нелинейных колебаний, Москва 1963.

3. N. Kauderer, Nichtlinear Mechanik, Berlin 1958.

\section{DAO ĐộNG THAM SỐ CÁC Hệ CƠ HỌC NHIỀU BẬC TỰ DO GÂY NÊN BỞI TÁC DỰG CỪA LỰC Đị̣̂N TỪ}

Công trình này là sự tiếp tục của công trình đã được công bố [1]. Trong công trình này kết quả nghiên cứu dao động của hệ cơ học vởi $n$ bậc tự do và của dầm khi chúng chịu tác dụng với lực điện từ của dao động kích động tham số tương tự như trong [1] dao động tham số được khảo sát có tần số bằng tần số dao động trong khung điện. Xác định biên độ dao động và nghiên cứu ổ định của chúng. 